\title{
Development of a Pedestrian Crossing Navigation System for a Visually Impaired Person Using MY VISION
}

\author{
Kohei Kitagawa \\ Department of Mechanical and Control Engineering, Kyushu Institute of Technology, Sensui-cho Tobata-ku \\ Kitakyushu, 804-8550, Japan \\ Seiji Ishikawa, Joo Kooi Tan \\ Department of Mechanical and Control Engineering, Kyushu Institute of Technology, Sensui-cho Tobata-ku \\ Kitakyushu, 804-8550, Japan \\ E-mail: \{kitagawa-kohei,etheltan\}@ss10.cntl.kyutech.ac.jp \\ http://lab.cntl.kyutech.ac.jp/ etheltan/
}

\begin{abstract}
In this paper, we propose a system for a visually impaired person to cross a pedestrian crossing safely by the employment of the state of a traffic light and the remaining distance to the other side obtained from the analysis of MY VISION images. The traffic light at a pedestrian crossing is detected by a discriminator using HOG (Histograms of Oriented Gradients) features and Random Forest. The effectiveness of the proposed method was verified by experiments.
\end{abstract}

Keywords: MY VISION, Visually Impaired, HOG, Random Forest, Traffic light, Pedestrian crossing behavior.

\section{Introduction}

The 2011 Ministry of Health, Labor and Welfare report writes that there are approximately 310,000 people in Japan who have visual impairment. Looking at the world, it is predicted that the number of visually impaired people worldwide will triple within the next 40 years ${ }^{1}$.

Accidents of visually impaired people are not uncommon, and there is a survey result that about $42 \%$ of the subjects experienced accidents with injuries while walking outdoors within the past 5 years, especially against automobiles ${ }^{2}$. It is easy to imagine that accidents with cars may often happen at an intersection, which may be one of the difficult points for a visually impaired person to cross.

It is based on Braille blocks and acoustic signs that a visually impaired person finds an intersection and judges the timing of crossing it. However, neither all intersections are installed Braille blocks, nor they have acoustic instruments. Therefore, it is necessary to have a navigation system that recognizes and analyzes (pedestrian) traffic lights, so that a visually impaired person may cross the intersection safely.

As a traffic light detection method, a method using template matching and a method utilizing position information by GPS or a map have been proposed ${ }^{3-5}$. However, all the studies are limited to the detection of traffic lights and they do not analyze possible timing to cross. However, in practice, it is vital for a visually impaired person to receive the information on the timing to cross. It should be realized by the analysis of traffic light images, once it is detected.

In this paper, we propose a method of detecting a pedestrian traffic light at a pedestrian crossing by MY VISION and analyzing it if one can walk into the crossing now. MY VISION is a self-virtual viewpoint employing a body (or a head) mounted camera. It can be a virtual eye of a visually impaired person or the third eye of a person who is careless ahead. In the proposed method, 
the traffic light is detected employing the images captured by a camera attached to the head of a person. The HOG feature is used for describing a traffic light. The traffic light is recognized by a Random Forest classifier, which is a multi-class classifier. Then pattern analysis is performed with the traffic light in the image sequence, and the possibility is judged if one may cross the frontal pedestrian crossing.

\section{Traffic Light Detection}

In this section, we describe detection of traffic lights, extraction of target object candidate regions, feature extraction using HOG features, and learning and identification by Random Forest.

\subsection{Extraction of a signal candidate area}

In the proposed method, instead of searching for a traffic light in a whole input image, it is searched in the upper half of the input image after converting it to a HSV image, which is close to human color recognition ${ }^{6}$. A candidate area of a traffic signal is extracted by searching for the signal color (red or green) in the upper half image. The reason the upper half of an image is considered is that the traffic light normally exists only in the upper half area of a self-virtual viewpoint image.

Once the candidate areas of a traffic signal are extracted, the areas receive expansion processing, since the color area of the traffic light may be extracted smaller than it actually is. After the expansion, labeling is performed to the expanded areas and those noisy areas are removed using a certain threshold value. Thus a candidate area of a traffic light is obtained.

\subsection{Histogram of Oriented Gradients (HOG)}

The HOG feature ${ }^{7}$ is used in the proposed method as a feature for detecting a traffic light, as it is robust to lighting changes, misalignment, and rotation. The gradient direction is quantized in four directions to accommodate angular changes.

\subsection{Learning and identification by Random Forest}

In the method, Random forest ${ }^{8}$, which is capable of multiclass discrimination, is employed for a classifier. Random forest is possible to learn and identify multiple target objects at the same time. By using it, we can improve generalization ability for unknown data in preparation for future multiclass classification..

\section{Crossing Analysis}

In this section, the method is explained for analyzing the situation of a traffic light and determining the timing of crossing. The analysis is performed based on the pedestrian crossing behavior principle hypothesis 9 .

\subsection{The hypothesis on pedestrian crossing behavior principle}

The hypothesis on pedestrian crossing behavior principle has been proposed, which gives insight into the relationship between pedestrian signals and pedestrian behavior.

The following four empirical patterns are considered in the hypothesis based on the situation of the traffic light

1. Experience on the change of the light from red to blue: The case where the length of the blue light is perceptible and it is considered to be the safest to cross.

2. Experience on only blue light display: The case where the length of the blue light display cannot be perceived and it is unknown when it will turn to a blinking signal. Depending on the walking speed of a person, it may be dangerous.

3. Experience on the change from blue to blinking blue: This is the case where the length of the blue blinking time is perceptible, and a person on cross can finish crossing.

4. Experience on only blinking blue light. The length of the blue blinking time cannot be perceived, and it is not possible to cross.)

Based on this crossing behavior principle hypothesis, pattern identification and analysis of a traffic light are performed.

\subsection{Input of walking status}

In the proposed method, crossing analysis takes the walking condition of a user into account. The information on what kind of walking situation a user is in is collected in advance.

The followings are two patterns of walking situation with a user.

A) Walks normally.

B) Cannot walk normally. 
Once either of the above walking conditions is entered before using the system, it is taken into consideration when the pattern 2 in 3.2 is identified.

\subsection{Discrimination of traffic lights}

This section describes how to discriminate the color and blinking of a traffic light.

\subsubsection{Color discrimination}

The color of the traffic light is determined based on the HSV model. The color of the signal (red/blue) is identified by setting certain thresholds on $\mathrm{H}, \mathrm{S}$ and $\mathrm{V}$ values and counting the number of pixels of each color in the search window.

\subsubsection{Identification of blinking signal}

The blinking cycle of the traffic light is used to determine whether the signal is blinking or it is the frame a traffic light cannot be detected. The blinking cycle of the traffic light is 0.5 seconds in Japan. If this cycle is satisfied, it is determined as a blinking signal, and if it is any other cycle, it is not detected.

\subsection{Pattern discrimination on traffic signals}

Based on the hypothesis on pedestrian crossing behavior principle explained in Section 3.1, pattern discrimination is performed in the sequence of video frames. There are four signal conditions: (a) red to blue, (b) blue, (c) blue to blue blinking, and (d) blue blinking.

\subsection{Cross judgment}

The proposed method determines whether a person is crossing a pedestrian crossing or before crossing it, and calculates approximate crossing distance. Considering that a pedestrian crossing exists in the lower half area of an image, the input image is processed only the lower half.

The pedestrian crossing image is processed in the following way. Image noise is removed by preprocessing using a Gaussian filter (Fig. 1(1)). Then it is binarized by the discriminant analysis ${ }^{10}$ to make it less insensitive to outdoor weather change.

After performing edge extraction with a Sobel filter, a probabilistic Hough transform ${ }^{11}$ is applied to the edge image to detect straight lines composing a pedestrian crossing. This process is faster than normal Hough transform.

Finally, based on the obtained straight lines (Fig. 2 (5)), the number of lines is counted and the length of the crossing is calculated (Fig.2(6)).

\subsection{Judgment on crossing possibility}

Whether to cross or not is judged based on the hypothesis on crossing behavior principle. As shown in Fig.2, the signal pattern is classified into 6 patterns and pattern identification is performed.

The output is done by voice. (For example, "The traffic signal will change soon.”, "About 3 meters remaining.”)

\section{Experimental Results}

An experiment was conducted to examine the performance of the proposed method. The used camera is a Sony FDR-X3000. It is mounted on the head of a subject. Five videos were evaluated for the situation analysis including traffic light detection. The traffic signal situation was analyzed including stationary signal and blinking signal discrimination, the judgment on crossing possibility. The pedestrian crossing distance was also estimated.

The results are shown by $F$-values. The average $F$ value of traffic light detection was 0.89 . The average $F$ values of red, blue and blinking signals detection were $0.87,0.89$ and 0.94 , respectively. Finally, the correct answer rate for crossing possibility judgment was 0.91 , and the relative error of the distance estimation on a

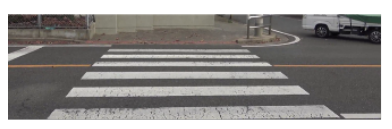

(1)Input

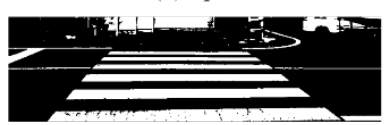

(3)Binarization

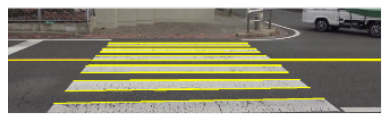

(5)Line detection

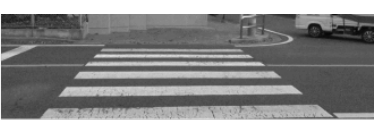

(2)Noise Reduction

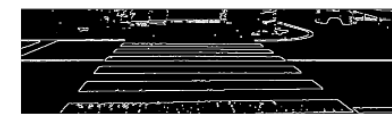

(4)Edge Extraction

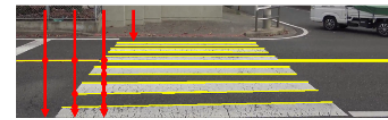

(6)Calculation
Fig. 1. Flow of the pedestrian crossing distance calculation: (1) Input image, (2) noise reduction, (3) binarization, (4) edge detection, (5) line detection, and (6) calculation of the distance. 


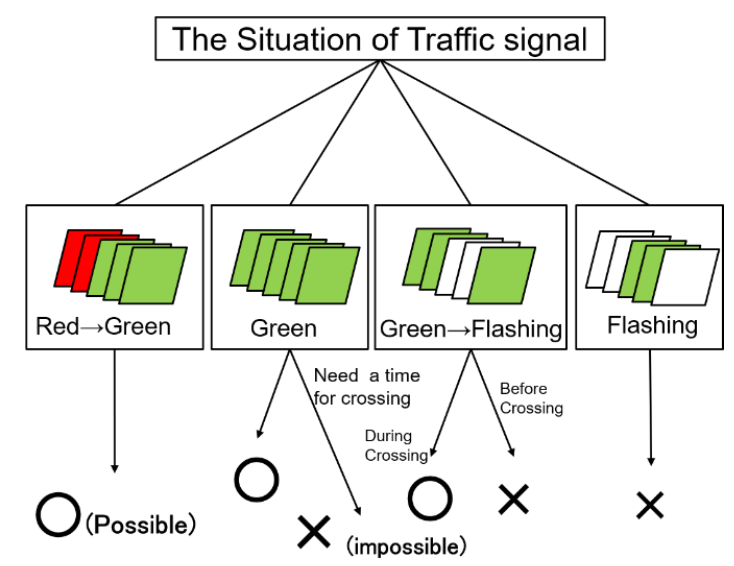

Fig. 2. Pattern identification: Patterns are defined using Pedestrian Crossing Behavior Principle Hypothesis, user status and judgment during crossing.

pedestrian crossing was $25.4 \%$. Fig. 3 shows a part of the experimental video.

\section{Discussion}

In the traffic light detection experiment using $\mathrm{HOG}$ features and Random Forest, the average $F$-value was 0.89 , which was an acceptable detection result. The reason of this good result may be the selection of 4 bins, which could absorb varying inclination of the line segments composing a traffic light.

Regarding the analysis of the traffic light, the signal detection including the blinking signal showed satisfactory results.

\section{Conclusion}

We proposed a method of detecting and analyzing pedestrian traffic lights from MY VIOSIN images. The effectiveness of the proposed method was verified by experiments. We plan to analyze not only traffic lights but also other kinds of information for a visually impaired person to walk safe in an outdoor environment, and consider developing a navigation system that is more appropriate to the actual environment.

\section{References}

1. Bourne, R. R. A., et al., Magnitude, temporal trends, and projections of the global prevalence of blindness and distance and near vision impairment: a systematic review and meta-analysis, The Lancet Glob Health 5(9), 2017, e888-e897.

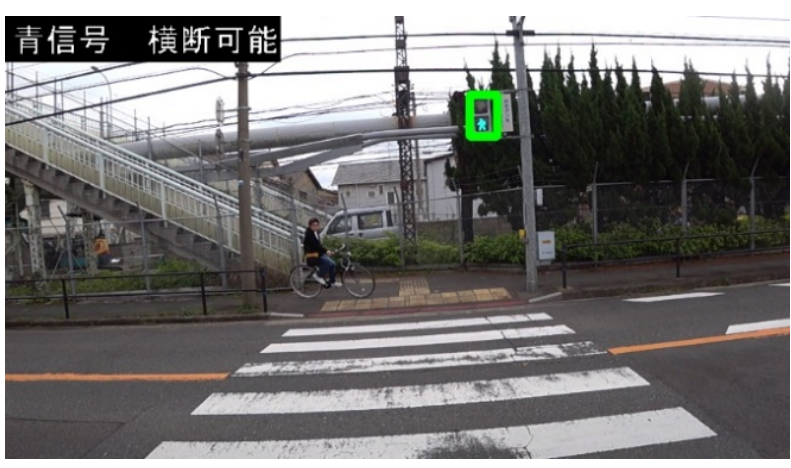

Fig. 3. Part of the experimental video.

2. S. Abe, N. Hashimoto, Survey on walking for the visually impaired, Bulletin of Hachinohe Institute of Technology $\mathbf{2 4}$, 2015, pp. 81-92. (In Japanese)

3. M. Imano, T. Hosokawa, et al., Research on autonomous movement of guide robots that guide visually impaired people, 2018 Tohoku chiku wakatekenkyusha happyoukai, 2018. (In Japanese)

4. Y. Baba, Y. Sakai et al., Real-time recognition of pedestrian signals from moving images, Proc.77th National Convention of IPSJ 1, 2015, pp.543-544. (In Japanese)

5. N. Fairfield, C. Urmson, Traffic light mapping and detection, 2011 IEEE International Conference on Robotics and Automation (ICRA), 2011, pp.5421-5426.

6. T. Kumano, J. K. Tan, H. Kim, S. Ishikawa, Traffic signs and signals detection employing the My Vision system for a visually impaired person, International Journal of Research and Surveys, ICIC Express Letters, partB: Applications 7(2), 2016, pp.385-391.

7. N. Dalal, B. Triggs, Histograms of oriented gradients for human detection, Proc. of IEEE Conference on Computer Vision and Pattern Recognition 1, 2005, pp.886-893.

8. L. Breman, Random forests, Machine Learning 45(1), 2001, pp.5-32.

9. K. Hatoyama, S. Sugimori, Analysis on Pedestrian Behavior Considering Spaces before the Crosswalk, Proceedings of infrastructure planning 30, 2004, p.171. (In Japanese)

10. N. Otsu, A threshold selection method from gray-level histograms, IEEE Transactions on systems, man, and cybernetics 9(1), 1979, pp.62-66.

11. N. Kiryati, Y. Eldar, A. M. Bruckstein, A probabilistic Hough transform, Pattern Recognition 24(4), 1991, pp.303-316. 